\title{
SHEAR BOND STRENGTH OF VARIOUS DENTAL CERAMICS TREATED WITH TWO TECHNIQUES OF SURFACE TREATMENT AFTER THERMOCYCLING
}

\author{
Raiesa Mohamed Hashem*
}

\begin{abstract}
Aim: The goal of this work was to compare the shear bond strength of various ceramics bonded to tooth structure following thermocycling using a traditional versus a simplified Surface Treatment.

Materials and methods: Thirty removed human mandibular molar teeth were separated into three groups $(n=10)$ based on the type of ceramic material employed (zirconia reinforcing lithium silicate (Celtra duo), Polymer infiltrated glass ceramic (Vita enamic), and lithium disilicate ceramic (IPS E Max cad)). Each group was divided into two categories based on the surface treatment process $(n=5)$ The classic approach (hydrofluoric acid with silane coupling agent) was used in Subgroup I, whereas the simplified method was applied by using Monobond Etch \& Prime(MEP) in Subgroup II. Ceramic discs were cemented to tooth structure using self-adhesive dual cure resin cement. Shear bond strength test (SBS) was carried out using universal testing machine. The data was obtained, processed, and statistically analyzed.
\end{abstract}

Results: It was found that a significant higher SBS mean values recorded for conventional technique in IPS Emax cad and Celtra duo group $(12.57 \pm 0.51,11.46 \pm 0.51 \mathrm{MPa})$ than that in simplified technique $(11.41 \pm 0.36,7.32 \pm 0.75)$ respectively. While in Vita Enamic group there was significantly higher SBS mean values recorded for the simplified technique (10.83 \pm 0.17$)$ using MEP than conventional technique $(8.24 \pm 0.46)$ using hydrofluoric acid with silane coupling agent.

Conclusion: Vita Enamic showed better results using Monobond Etch \& Prime surface treatment than HF acid and silane. While For Emax and celtra due HF acid and silane surface treatment has shown better results.

KEYWORDS: Surface treatment, Ceramics, Shear bond strength, Simplified technique.

\section{INTRODUCTION}

All ceramic restorations had a higher bond strength than non-retentive restorations as veneers ${ }^{1}$.
For improving bond strength better analysis of the internal structure of restorative materials, as well as optimal cement selection and surface treatment. ${ }^{2}$.

* Fixed Prosthodontics Department, Faculty of Dentistry,Minia University, Egypt 
Resin-based cement is the material of choice for luting cement in ceramic restorations. ${ }^{3}$. Resin cement has a low solubility in oral situations compared to other luting cements and adheres well to a range of dental or ceramic surfaces. ${ }^{3,4}$.

The success of all-ceramic restorations is dependent on the cementation processes, which are controlled by surface conditioning procedures, ceramic materials, and cementing chemicals. ${ }^{5-7}$ Different surface conditioning methods are necessary because varying kinds of ceramics have different chemical compositions. ${ }^{4,8}$

Conditioning dental ceramics using hydrofluoric acid and silane provides increased chemical bonding by assisting contact with the ceramic due to the presence of bi-functional molecules. ${ }^{9}, 10$ Moreover surface pits are formed when hydrofluoric acid dissolves the glassy phase of ceramics. With the addition of silane, the wettability of the ceramic is increased, and covalent connections between the ceramic and cement are formed.

Despite its use, hydrofluoric acid has a number of disadvantages. As a result, skipping this step for such ceramics would be tremendously helpful, but only if a strong enough silane bond could be produced elsewhere. ${ }^{3,4}$

Recently; ammonium polyfluoride replaced $\mathrm{HF}$ and also it has silane in its composition so it integrate the two surface treatments and hence gives easier bonding procedure. However there are few researches about this technique

The purpose of this research is to evaluate the shear bond strength of various types of dental ceramics including lithium disilicate glass (IPS Emax CAD), zirconium-reinforced lithium silicate glass (Celtra Duo), and polymer infiltrated ceramic (Vita Enamic) cemented to tooth structure after various surface treatments using the traditional path of hydrofluoric acid etching plus silane coupling agent surface treatment and another surface treatment using Monobond Etch \& Prime.

\section{- Hypothesis}

The current study's premise was that varied surface treatment procedures would affect the shear bond strength of various ceramics to tooth structure.

\section{MATERIALS \& METHODS}

\section{- Ethical regulation}

- Teeth for this study were collected from Minia University's Faculty of Dentistry's outpatient clinic. Patients signed a consent form indicating that their teeth will be utilised in dentistry research. Teeth will not be utilised in any other research when the study is over, and they will be burned out.

\section{Sample Grouping}

- Thirty retrieved human maxillary molar teeth were divided into 3 categories $(n=10)$ based on the type of ceramic material used to assess and compare the shear bond strength of three different types of ceramic veneers, zirconia reinforcing lithium silicate ${ }^{*}$, polymer infiltrated glass ceramic $^{* *}$, and lithium disilicate ceramic ${ }^{* * *}$. Then, based on the surface treatment technique utilized, each group will be divided into two classes: conventional and simplified $(n=5)$.

\section{Teeth selection:}

- Thirty freshly removed human molars with no evidence of demineralization, white spot lesions, fractures, abrasions, or gaps were collected. The molars that were gathered were caries-free, and they were removed for periodontal purposes.

\footnotetext{
* Dentsply, Sirona, Germany

** VITA Zahnfabrik

$* * *$ Ivoclar Vivadent, Liechtenstein
} 
All teeth were checked under a magnifying loop at a magnification of $25 \mathrm{x}$ to ensure that they were free of cavities, restorations, and fractures. The ultrasonic scalers with $25 \mathrm{KHZ}$, nylon bristle brushes, and pumice with a low speed hand piece 30,000 RPM were utilized to clean the teeth from dirt and any soft tissues. The teeth were then preserved in distilled water until usage.

\section{Teeth mounting:}

- All the teeth were mounted in epoxy resin* using teflon cylinder mold (21 mm diameter $\mathrm{x}$ $25 \mathrm{~mm}$ high). Notches were prepared on the root of the teeth to enhance mechanical interlocking to epoxy resin. A sufficient amount of base and catalyst liquid of self-cured epoxy resin was prepared and put into the teflon mold according to the manufacturer's specifications. The roots of each tooth were placed in the center of the mold, covering the furcation zone, and held in place vertically by a piece of wax sheet created to keep the tooth in place until the epoxy resin had completely set.

\section{Teeth preparation}

- A depth preparation bur** was used to create depth-orientation grooves on the buccal surface of the teeth $(0.5 \mathrm{~mm}$ in depth). After that, the samples were prepared to generate a smooth enamel surface region of about $5 \mathrm{~mm}$ in diameter, which was utilised to luting the ceramic discs to the middle third of the facial surface without going past the depth-orientation grooves.

\footnotetext{
* Acrostone cold curespecial tray material,england

** Dentsply, Maillefer, Switzerland

*** IsoMet 4000 microsaw, buehler, Lake bluff, IL, USA

***** Programat P310; Ivoclar Vivadent

$* * * * *$ Ultradent $^{\mathrm{TM}}$ Products, Inc, USA

$* * * * * *$ Ultradent $^{\mathrm{TM}}$ Products, Inc, USA

$* * * * * * *$ Ivoclar Vivadent, Liechtenstein
}

\section{Cutting and crystallization of the ceramic discs:}

- A rectangular cross sectional shaped ceramic blocks were prepared to be cylindrical cross sectional shaped with a diameter of five millimeter. After that milling of discs was made into a thickness of $2 \mathrm{~mm}$ in a precision saw machine ${ }^{* * *}$.

- In a ceramic furnace ${ }^{* * * *}$, Celtra Duo and IPS e.max CAD discs were crystalized according to the manufacturer's requirements. It was not necessary to burn the vita enamic discs.

\section{Surface treatment of the ceramic discs:}

- Surface treatment of ceramic discs using conventional technique

1. Hydroflouric acid of concentration $9.6 \%^{* * * * * *}$ was used for etching the bonding surfaces of the ceramic discs for 20 seconds. Then washed with air/water spray for 30 seconds and dried for another10 seconds.

2. The surfaces were then treated with a puddle coat of silane coupling agent ${ }^{* * * * *}$, applied with the syringe's Black MiniTM brush tip, and allowed to react for 60 seconds before being air-dried without cleaning.

- Surface treatment of ceramic discs using the simplified technique

- Monobond Etch \& Prime P $^{* * * * * * *}$ was applied on the surfaces that were previously prepared for bonding by using micro brush, for 20 seconds and afterwards are allowed to react for 40 seconds before being washed and airdried for 10 seconds. 
- Surface treatment of tooth surface before cementation.

- With 37 percent phosphoric acid*, the tooth surfaces were etched for 20 seconds. After that, the surfaces were cleaned with water and blotted dry.

- After that Tetric N-Bond** was applied by a micro brush and dried for 10 seconds then light-cured ${ }^{* * *}$ for another 20 seconds.

- Cementation of ceramic discs to tooth structure with resin cement:

- On the prepared buccal tooth surface, a thin coating of self-adhesive dual-cure resin cement $^{* * * *}$ was applied. each disc was positioned on its associated tooth and held in place. For 6 minutes, a constant weight of $3 \mathrm{~kg}$ was applied parallel to the long axis of each disc to avoid rebounding during cementation. The light cure apparatus was used to cure the cement for 2 seconds. A sharp hand scaler ${ }^{* * * * *}$ was used to remove the extra cement from the edges. The cement was given a light cure for 20 seconds.

\section{- Preparation for testing}

- Before testing, all samples (cemented ceramics discs on the teeth surfaces) were incubated for 24 hours in distilled water chambers at $37^{\circ} \mathrm{C}$ and $100 \%$ humidity. After that all samples were thermocycled ${ }^{* * * * * *}$ for 5000 cycles from $5^{\circ} \mathrm{C}(20 \mathrm{~s})$ to $55^{\circ} \mathrm{C}$. The time it took to switch between baths was 10 seconds.

- Shear Bond Strength Testing of the samples.

- The shear bond strength test was performed using universal testing machine $\mathrm{e}^{* * * * * * *}$, and the data was collected using computer software ${ }^{* * * * * * * *} \mathrm{~A}$

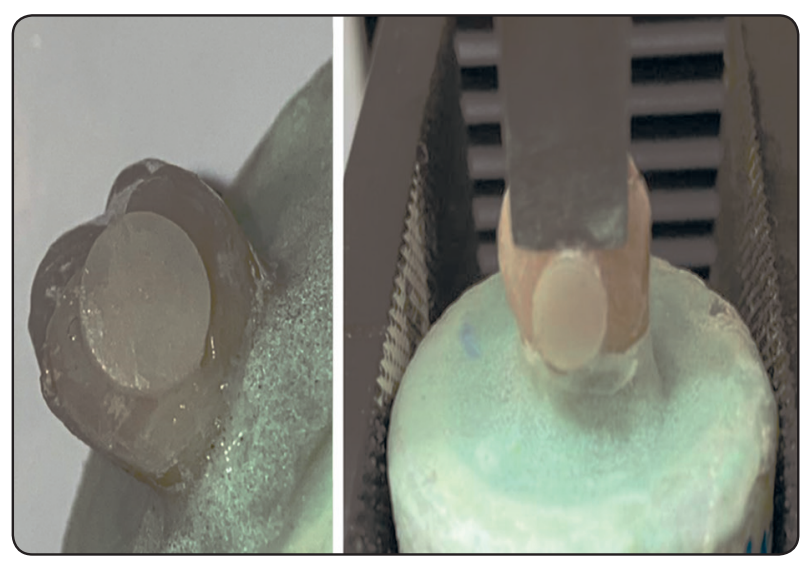

Fig. (1) Cemented ceramic disc on tooth surface before and during measurement.

chisel-shaped blade with a $0.6 \mathrm{~mm}$ thick edge was used to apply the shearing force in a compressive mode of load. The specimens were loaded at a cross-head speed of $0.5 \mathrm{~mm} / \mathrm{min}$ with a 5000 Newton load cell until the bond ruptured and the shear force was calculated. Newtons were used to compute the force. Converting Newtons to Megapascals was used to calculate shear bond strength (MPa)

\section{- Mode of cementation failure analysis}

After debonding all teeth surfaces and ceramic discs were viewed using USB Digital microscope with a built-in camera connected with an IBM compatible personal computer using a fixed magnification of 65 . Three failure types were expected to be observed. Adhesive (when the fracture occurred at the tooth, resin cement/ceramic interface), cohesive (when remnants of resin cement on the tooth and remnants on the ceramic disc and Mixed failure (when a combination of adhesive and cohesive failures).

\footnotetext{
* META BIOMED,KOREA

** Ivoclar Vivadent, Liechtenstein

*** Demi Plus LED Light Curing System, Kerr, USA

**** Ivoclar Vivadent, Liechtenstein

***** Carl Martin GmbH, Germany

****** THE 100 SD Mechatronic thermocycler Germany

******** Instron, model 3345, England

******** Bluehill software version 3
} 


\section{RESULTS}

\section{Statistical method}

Version 25 of the SPSS application (Statistical Package for Social Sciences). The collected data was statistically analysed using this programme. For parametric (normally distributed) quantitative data, descriptive statistics were calculated using the mean, standard deviation (SD), and minimum and maximum ranges. The Shapiro Wilk test was used to disperse the data. The influence of material, method, and their interaction on shear bond strength was investigated using a two-way ANOVA test. The One-Way ANOVA test was used to analyze parametric quantitative data between more than two groups, followed by post hoc analysis between each two groups. The Independent Samples T test was used to compare the parametric quantitative data of the two groups. The significance level was set at 0.05. (P value 0.05).

- The results of Emax and Celtra duo revealed a significant higher SBS mean values recorded for conventional technique $(12.57 \pm 0.51 \mathrm{MPa}$, $11.46 \pm 0.51)$ than simplified technique $(11.41 \pm$ $0.36,7.32 \pm 0.75)$ respectively. While Vita Enamic, The results revealed significantly higher
SBS mean values recorded for the simplified technique $(10.83 \pm 0.17)$ using MEP than conventional technique $(8.24 \pm 0.46)(\mathrm{HF}+\mathrm{S})$ as shown in figure (2).

- The results revealed that the shear bond strength mean values obtained for samples cemented on E-max $(12.57 \pm 0.51)$ using the conventional way of surface treatment were higher than those cemented on Celtra duo (11.46 \pm 0.51$)$ and Vita Enamic (7.32 \pm 0.75$)$. According to the statistical test, there significant difference between the Emax, Celtra duo and the Vita Enamic group. (Pvalue <0.05).

- As regard the simplified technique, the results shows that shear bond strength mean values recorded for the samples which were cemented using simplified technique of surface treatment on Emax (11.41 \pm 0.36$)$, were higher than that cemented on Vita Enamic $(10.83 \pm 0.17)$ and Celtra duo (7.32 \pm 0.75$)$. The statistical test illustrated that a non-significant difference between Emax and vita enamic as indicated by one way ANOVA test ( $P$ value $<0.05)$. While there was a significant decrease in shear bond strength in celtra duo group compared with Vita Enamic and Emax groups.

TABLE (1): Comparisons of shear bond strength between different materials and different techniques.

\begin{tabular}{|c|c|c|c|c|c|c|}
\hline \multirow{2}{*}{ Technique } & \multirow{2}{*}{$\begin{array}{c}\text { E-Max (I) } \\
\mathrm{N}=5\end{array}$} & \multirow{2}{*}{$\begin{array}{c}\text { ZLS (II) } \\
\mathrm{N}=5\end{array}$} & \multirow{2}{*}{$\begin{array}{c}\text { Vita Enamic (III) } \\
\mathrm{N}=5\end{array}$} & \multicolumn{3}{|c|}{ P value (between different materials) } \\
\hline & & & & I vs II & I vs III & II s III \\
\hline \multirow{2}{*}{$\begin{array}{l}\text { Conventional surface } \\
\text { treatment }\end{array}$} & $(12.01-13.15)$ & $(10.93-12)$ & $(7.78-8.99)$ & \multirow{2}{*}{$0.011 *$} & \multirow{2}{*}{$<0.001 *$} & \multirow{2}{*}{$<0.001 *$} \\
\hline & $12.57 \pm 0.51$ & $11.46 \pm 0.51$ & $8.24 \pm 0.46$ & & & \\
\hline \multirow{2}{*}{ Simplified technique } & $(10.99-11.82)$ & $(6.46-8.23)$ & $(10.62-11)$ & \multirow{2}{*}{$<0.001 *$} & \multirow{2}{*}{0.196} & \multirow{2}{*}{$<0.001 *$} \\
\hline & $11.41 \pm 0.36$ & $7.32 \pm 0.75$ & $10.83 \pm 0.17$ & & & \\
\hline $\begin{array}{l}\text { P value (between different } \\
\text { Techniques) }\end{array}$ & $<0.001 *$ & $<0.001 *$ & $<0.001 *$ & & & \\
\hline
\end{tabular}

For quantitative findings, a one-way ANOVA test comparing the three materials in each procedure was used,followed by a post-hoc analysis between the two materials.

Comparing the two procedures using a T test for quantitative data in each material *: Various individuals' samples A T test was employed to compare quantitative findings from the two techniques in each item. With a P value of 0.05 , there is a high degree of significance. 


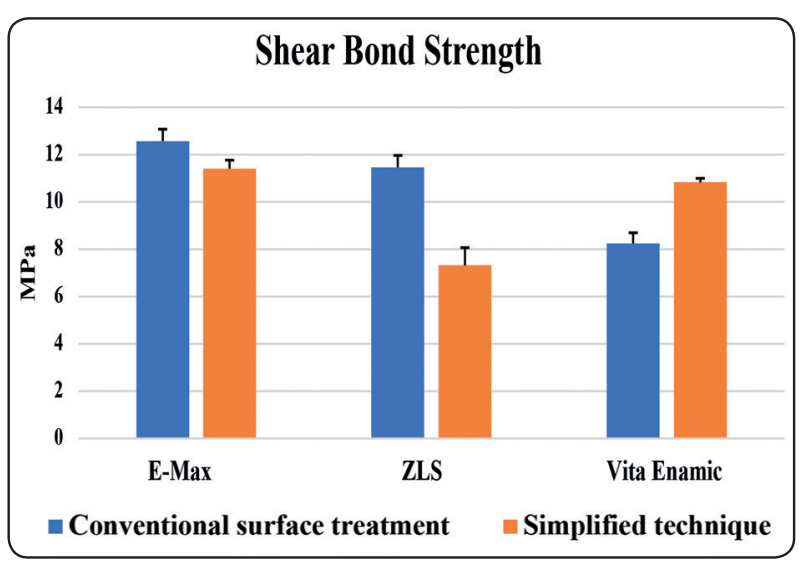

Fig. (2): Histogram showing shear bond strength between different materials and different techniques

\section{Failure pattern analysis results}

Emax samples cemented by the conventional technique showed mixed type of failure in 4 samples while one sample showed cohesive failure, on the other hand all samples in the simplified technique showed mixed type of failure. Regarding Celtra duo, all cemented samples using the conventional surface treatment showed mixed type of failure, whereas the simplified technique showed mixed failure in three sampled while two samples showed adhesive failure. All samples of vita enamic using the simplified surface treatment showed mixed type of failure, while three samples in conventional surface showed mixed failure and two samples showed adhesive failure

\section{DISCUSSION}

The cementation processes, which are connected to the kind of ceramic materials, cementing agents, and surface-conditioning techniques ${ }^{4,7}$, are crucial to the effectiveness of all-ceramic restorations. On the surfaces of various types of ceramics with various chemical compositions, several surfaceconditioning processes are applied., ${ }^{4,8}$

In vitro investigations are required to establish the surface treatment that will result in the maximum bond strength as ceramic materials become more widely employed in daily practise. As a result, ceramic materials with various chemical compositions and distinct clinical indications were investigated in this study.

Emax is a lithium disilicate glass-ceramic material that utilized in all-ceramic restorations. It is recommended for anterior or posterior crowns, inlays, onlays, implant crowns, or veneers. The CAD/ CAM material was initially only available as a substructure material, with a lower translucency than other high-strength ceramic core materials. ${ }^{11}$ Celtra Duo is a lithium-silicate ceramic enhanced with zirconium oxide and containing $10 \%$ high dispersion zirconia. When zirconia is present, a phosphate monomer should be used to make a chemical bond with the resin adhesive, which may entail the use of a di-functional primer that can bind to both silica and zirconia phases. ${ }^{12,13}$ Vita Enamic is a polymerinfiltrated ceramic network (PICN) material composed of an 86 percent dominant network reinforced by a 14 percent acrylic polymer network, with both networks entirely permeating each other. ${ }^{13}$

As a result, HF is commonly utilized to produce mechanical attachment to ceramic, while silane promotes chemical bonding by permitting contact with the ceramic due to bi-functional molecules. ${ }^{14,15}$

For the following reasons, the HF etching stage of the ceramic restoration procedure should be avoided: (1) Hydrofluoric acid is very toxic and has been linked to a variety of serious health problems. ${ }^{16}$. (2) Etching silica-based ceramics using hydrofluoric acid has been reported to result in the formation of insoluble silica fluoride salts on the surface $^{3,17}$. The bonding strength of the resin may be weakened by the remaining by-products ${ }^{18}$ (3) Ammonium polyfluoride has been shown to be more biocompatible and safer than HF.

As a result, eliminating this step for certain ceramics would be extremely helpful, but only if a strong enough silane bond could be achieved. ${ }^{3}$

The use of self-etching ceramic primers (SECP) for ceramic surface treatment has the potential 
to simplify the clinical procedure, increase the likelihood of a structurally solid and appealing ceramic repair, and assure laboratory and clinical operator safety.

MEP (Monobond Etch \& Prime) is a revolutionary one-bottle method that replaces hydrofluoric acid with ammonium polyfluoride and silane. Despite its name, self-etching ceramic primer (SECP) should be washed with water after usage. This method streamlines the bonding process by etching and priming glass-ceramics in one step while maintaining the ceramic's adhesive capabilities. ${ }^{19}$

Compared to $\mathrm{HF}+\mathrm{S}$, MEP etching produces less roughness and a shallower etching pattern. Meanwhile, the MEP silane system (based on trimethoxypropyl methacrylate) leaves a chemically bonded thin layer of silane on the treated surface after complete washing and drying. Although the mechanism of action of MEP is unknown, elemental analysis revealed that following pretreatment with MEP, some fluorine residue is present on the surface.$^{20}$ The material's interaction with the glassy component produces insoluble silica-fluoride salts, which are left as residue or deposit on the surface, explaining the presence of $\mathrm{F}$ ions residue ${ }^{21}$, or because $F$ is trapped within the silane layer left on the repair's surface. The cleaning process used after the etching determines this. Other cleaning procedures might produce different results ${ }^{22}$. The clinical relevance of this $\mathrm{F}$ residue is currently unknown, and more research is needed.

The results of this investigation showed that the conventional strategy yield significantly larger SBS mean values than the simplified technique in the lithium di-silicate (LDC) Emax. A variety of investigations led to this conclusion. ${ }^{20,23-27 .}$

The greater surface area and higher surface roughness induced by the usual way of surface treatment can explain the rise in bond strength values when utilising an etching step with $\mathrm{HF}+\mathrm{S}$. Rania and Bayoumi (2019) ${ }^{28}$ The surface roughness of lithium disilicate and SBS were shown to have a substantial positive association, according to their study.

Hydrofluoric acid, which creates microporosities on the glass-ceramic surface, increases surface area, and aids mechanical interlocking, can also be used to demonstrate this. In the glass ceramic-resin connection, silane works as a coupling agent, adsorbing onto the glass-ceramic surface and increasing chemical interaction. The use of silane appears to be critical for the bond's durability, according to many in vitro experiments..$^{29,30}$

In the case of Vita Enamic, the findings revealed that the simplified approach utilising MEP recorded much higher SBS mean values than the standard technique $(\mathrm{HF}+\mathrm{S})$. These are the outcomes of Murillo-Gómez and De Goes' research (2019). ${ }^{31}$ This didn't match the findings of previous investigations. ${ }^{32,33,34}$

This is due to the fact that the HF acid treatment appears to partially dissolve the Vita Enamic polymer and glassy phases. However, when subjected to stronger HF etching, which dissolves a higher portion of glassy phase, polymer structure existing on PIC is disclosed. This might mean that vigorous HF etching removes silicon from the surface of PICs, making resin cement bonding more difficult due to hindering the action of silane primers. As a result, the mildest etching process available should be used to treat PIC in order to roughen the surface while maintaining the majority of the silicon content..$^{35}$

MEP, on the other hand, exhibited smoother etching patterns and lower roughness values than any other HF etching procedure. ${ }^{35}$ this is due to the fact that instead of ordinary HF, this primer employs ammonium polyfluoride as an etching agent. In comparison to HF conventional methods, MEP may be regarded a less damaging option.

The Celtra duo's findings with zirconia reinforced lithium silicate (ZLS) showed that the conventional process had much higher SBS mean values than the semplified technique. This is due to 
the fact that when a glass-ceramic surface is etched with $10 \% \mathrm{HF}$, the glass matrix dissolves more fully, exposing the crystalline structure ${ }^{36}$ As a result, topography with a larger total contact area than a smooth surface is produced ${ }^{37}$. This impact may have been exacerbated by the employment of the silane bonding agent and its chemical connections. ${ }^{38}$

In terms of the conventional technique $(\mathrm{HF}+\mathrm{S})$, MSBS had the highest mean values for samples on Emax, followed by celtra duo, while Vita Enamic were the lowest with a significant difference. The results were in accordance with Della Bona and associates $(2003)^{6}$, Frankenberger et al $(2015)^{33}$, Peuet al (2016) ${ }^{30}$, and El-Damanhoury et al (2017) ${ }^{20}$.

The enhanced bonding efficacy of resin luting materials to lithium disilicate can be attributed to a better chemical interaction between the hydrophobic resin and the Emax surface rather than mechanical interlocking to the rough surface. The variances in results might be explained by differences in surface topography, surface energy, chemical composition, and responsiveness to different surface treatment processes.

The idea that different surface treatment procedures will alter the shear bond strength of the chosen ceramic materials was partially rejected based on the findings of this investigation.

\section{CONCLUSION}

The Monobond Etch \& Prime surface treatment produced better results for Vita Enamic than HF acid and silane. While HF acid and silane have proved to improve hear bond strength in Emax and Celtra.

\section{REFERENCES}

1. Biacchi GR, Mello B, Basting RT. The endocrown: An alternative approach for restoring extensively damaged molars. J Esthet Restor Dent. 2013;25(6):383-390.

2. Dejak B, Mlotkowski A. Three-dimensional finite element analysis of strength and adhesion of composite resin versus ceramic inlays in molars. J Prosthet Dent. 2008;99(2): 131-140.
3. Kramer N, Lohbauer U, Frankenberger R. Adhesive luting of indirect restorations. Am J Dent. 2000;13(4):60-76.

4. de CARVALHO RF, MARTINS MEMN, de QUEIROZ JRC, LEITE FPP, Ozcan M. Influence of silane heat treatment on bond strength of resin cement to a feldspathic ceramic. Dent Mater J. 2011;30(3):392-397.

5. Kramer N, Lohbauer U, Frankenberger R. Adhesive luting of indirect restorations. Am J Dent. 2000;13(4):60-76.

6. Bona A Della, Anusavice KJ. Microstructure, composition, and etching topography of dental ceramics. Int J Prosthodont. 2002;15(2):159-167.

7. Matinlinna JP, Lassila LVJ, Özcan M, Yli-Urpo A, Vallittu PK. An introduction to silanes and their clinical applications in dentistry. Int J Prosthodont. 2004;17(2):155-164.

8. Özcan M, Vallittu PK. Effect of surface conditioning methods on the bond strength of luting cement to ceramics. Dent Mater. 2003;19(8):725-731.

9. Elbasty R, Taymour M. Assessment of resin -ceramic bond strength under various $\mathrm{pH}$ conditions for different ceramic materials subjected to two surface treatment protocols. Egypt Dent J. 2019;65(1):667-679.

10. Nogueira IDO, Gomes F, Magno B, Masterson D, Pereira T, Maia C. Does the application of an adhesive layer improve the bond strength of etched and silanized glass ceramics to resin-based materials ? A systematic review and meta-analysis. J Prosthet Dent. 2020;123(2):204-214.

11. Fasbinder D, Dennison J, Heys D, Neiva G. A Clinical Evalıation of Chairside Lithium Disilicate CAD/ CAM Crowns: A Two-Year Report. J Am Dent Assoc. 2008;141(2):10-14.

12. Aboushelib MN, Sleem D. Microtensile bond strength of lithium disilicate ceramics to resin adhesives. J Adhes Dent. 2014;16(6):547-552

13. Zimmermann M, Mehl A, Reich S. New CAD/CAM materials and blocks for chairside procedures. Int J Comput Dent. 2013;16(2):173-181.

14. Kamada K, Yoshida K, Atsuta M. Effect of ceramic surface treatments on the bond of four resin luting agents to a ceramic material. J Prosthet Dent. 1998;79(5):508-513.

15. Roulet JF, Söderholm KJM, Longmate J. Effects of Treatment and Storage Conditions on Ceramic/Composite Bond Strength. J Dent Res. 1995;74(1):381-387.

16. Bertolini JC. Hydrofluoric acid: a review of toxicity. J Emerg Med. 1992;10(2):163-168. 
17. Monticelli F, Toledano M, Osorio R, Ferrari M. Effect of temperature on the silane coupling agents when bonding core resin to quartz fiber posts. Dent Mater. 2006;22(11):1024-1028.

18. Shimada Y, Yamaguchi S, Tagami J. Micro-shear bond strength of dual-cured resin cement to glass ceramics. Dent Mater J. 2002;18(5):380-388.

19. Román-Rodríguez JL, Perez-Barquero JA, GonzalezAngulo E, Fons-Font A, Bustos-Salvador JL. Bonding to silicate ceramics: Conventional technique compared with a simplified technique. J Clin Exp Dent. 2017;9(3):384-386.

20. El-Damanhoury HM, Gaintantzopoulou MD. Self-etching ceramic primer versus hydrofluoric acid etching: Etching efficacy and bonding performance. J Prosthodont Res. 2017;62(1):75-83.

21. Canay Ş, Hersek N, Ertan A. Effect of different acid treatments on a porcelain surface. J Oral Rehabil. 2001;28(1):95-101.

22. Bruzi G, Carvalho AO, Giannini M, Maia HP, Magne P. Post-etching cleaning influences the resin shear bond strength to CAD/CAM lithium-disilicate ceramics. Appl Adhes Sci. 2017;5(1):1-9.

23. Wille S, Lehmann F, Kern M. Durability of Resin Bonding to Lithium Disilicate and Zirconia Ceramic using a Selfetching Primer. J Adhes Dent. 2017;19(6):491-496.

24. Román-Rodríguez JL, Perez-Barquero JA, GonzalezAngulo E, Fons-Font A, Bustos-Salvador JL. Bonding to silicate ceramics: Conventional technique compared with a simplified technique. J Clin Exp Dent. 2017;9(3):384-386.

25. De Goes M, Murillo-Gómez F. Long-term bond strength of glass-ceramic treated with acid ceramic primer. Dent Mater. 2017;33(s1):e24.

26. Al-Harthi AA, Aljoudi MH, Almaliki MN, El-Banna KA. Laboratory study of micro-shear bond strength of two resin cements to leucite ceramics using different ceramic primers. J Contemp Dent Pract. 2018;19(8):918-924.

27. Scherer MM, Prochnow C, Venturini AB, et al. Fatigue failure load of an adhesively-cemented lithium disilicate glass-ceramic: Conventional ceramic etching vs etch \& prime one-step primer. Dent Mater. 2018;34(8):1134-1143.

28. Bayoumi R, El-Kabbany S, Gad N. Effect of Different Sur- face Treatment Modalities on Surface Roughness and Shear Bond Strength of Orthodontic Molar Tubes to Lithium Disilicate Ceramics. Egypt Dent J. 2019;65(1):641-656.

29. Sundfeld D, Correr-Sobrinho L, Pini NIP, et al. The effect of hydrofluoric acid concentration and heat on the bonding to lithium disilicate glass ceramic. Braz Dent J. 2016;27(6):727-733.

30. Peumans M, Valjakova EB, De Munck J, Mishevska CB, Van Meerbeek B. Bonding effectiveness of luting composites to different CAD/CAM materials. J Adhes Dent. 2016;18(4):289-302.

31. Murillo-Gómez F, De Goes MF. Bonding effectiveness of tooth-colored materials to resin cement provided by selfetching silane primer after short- and long-term storage. J Prosthet Dent. 2019;121(4):713.e1-e8.

32. Elsaka SE, Elnaghy AM. Mechanical properties of zirconia reinforced lithium silicate glass-ceramic. Dent Mater. 2016;32(7):908-914.

33. Frankenberger R, Hartmann VE, Krech M, et al. Adhesive luting of new CAD/CAM materials [Adhäsive Befestigung neuer CAD/CAM-Materialien]. Int J Comput Dent. 2015;18(1):9-20.

34. Campos F, Almeida CS, Rippe MP, De Melo RM, Valandro LF, Bottino MA. Resin bonding to a hybrid ceramic: Effects of surface treatments and aging. Oper Dent. 2016;41(2):171-178.

35. Murillo-Gómez F, Palma-Dibb RG, De Goes MF. Effect of acid etching on tridimensional microstructure of etchable CAD/CAM materials. Dent Mater. 2018;34(6):944-955.

36. Sato TP, Anami LC, Melo RM, Valandro LF, Bottino MA. Effects of surface treatments on the bond strength between resin cement and a new zirconiareinforced lithium silicate ceramic. Oper Dent. 2016;41(3):284-292.

37. Della Bona A, Shen C AK. Work of adhesion of resin on treated lithia disilicate-based ceramic. Dent Mater. 2004; 20(4):338-344.

38. Brentel AS, Ozcan M, Valandro LF, Alarça LG, Amaral R BM. Microtensile bond strength of a resin cement to feldpathic ceramic after different etching and silanization regimens in dry and aged conditions. Dent Mater J. 2007;23(1):1323-1331. 